\title{
Avaliação do Risco para Parto Prematuro Espontâneo pelo Comprimento do Colo Uterino no Primeiro e Segundo Trimestres da Gravidez
}

\author{
Risk Assessment for Spontaneous Preterm Delivery According to Cervical Length \\ in the First and Second Trimesters of Pregnancy
}

Mário Henrique Burlacchini de Carvalho, Roberto Eduardo Bittar, Midgley Gonzales, Maria de Lourdes Brizot, Marcelo Zugaib

\begin{abstract}
RESUMO
Objetivos: avaliar, no primeiro e segundo trimestres da gravidez, a correlação entre o comprimento do colo uterino e a ocorrência de parto prematuro espontâneo.

Métodos: em 641 grávidas do pré-natal geral, a medida do comprimento do colo uterino foi realizada em dois momentos: entre a $11^{a}$ e a $16^{a}$ e entre a $23^{a}$ e a $24^{a}$ semana da gravidez. A avaliação do colo uterino foi feita pela ultra-sonografia transvaginal, com a grávida em posição ginecológica e tendo realizado previamente esvaziamento da bexiga. O comprimento do colo uterino foi medido linearmente do seu orificio interno ao externo. A medida do comprimento do colo uterino foi correlacionada com a idade gestacional no momento do parto. Para comparação das médias do grupo de grávidas que tiveram parto prematuro e a termo utilizamos o teste t de Student. A sensibilidade, especificidade, taxa de falso-positivos, falso-negativos e acurácia para o parto prematuro foram calculadas para os comprimentos de colo menores ou iguais a $30 \mathrm{~mm}, 25 \mathrm{~mm}$ e $20 \mathrm{~mm}$.

Resultados: a medida do comprimento do colo uterino entre a $11^{a}$ e a $16^{a}$ semana de gestação não apresentou diferença significativa entre o grupo de parto prematuro e o grupo a termo (40,6 $\mathrm{mm}$ e 42,7 mm, respectivamente, $p=0,2459)$. A diferença entre os grupos, no entanto, foi significativa quando o colo foi medido entre a $23^{a}$ e a $24^{a}$ semana de gravidez $137,3 \mathrm{~mm}$ para o grupo que evoluiu com parto prematuro e 26,7 $\mathrm{mm}$ para o grupo a termo, $p=0,0001$, teste t de Student).

Conclusões: o comprimento do colo uterino entre a $11^{a}$ e a $16^{a}$ semana de gravidez não foi significativamente diferente entre as gestantes com parto prematuro ou a termo. No entanto, na $23^{a}$ e $24^{a}$ semana esta diferença foi significativa, podendo ser um método de predição da prematuridade.
\end{abstract}

PALAVRAS-CHAVE: Prematuridade. Gravidez normal. Comprimento do colo uterino.

\section{Introdução}

O parto espontâneo é precedido de alterações do colo uterino. O esvaecimento e o encurtamento do colo podem preceder o início das contrações uterinas detectáveis pela gestante ou pelo obste-

Setor de Baixo Peso Fetal da Clínica Obstétrica do Hospital das Clínicas da Faculdade de Medicina da Universidade de São Paulo

Correspondência:

Mário Henrique Burlacchini de Carvalho

Rua Barão do Bananal 640, apt ${ }^{\circ} 193$ - Pompéia

05024-000 - São Paulo - SP

e-mail: marioburlacchini@uol.com.br tra, representando risco para o parto prematuro. $\mathrm{O}$ achado de colo uterino curto na gestação precoce é fator de risco para o parto prematuro ${ }^{1-3}$.

A ultra-sonografia transvaginal é um ótimo método para avaliação do colo uterino na gestação. Permite a determinação do comprimento do colo uterino, bem como o diagnóstico de seu encurtamento ${ }^{4}$. Vários estudos correlacionaram o comprimento do colo uterino com risco para prematuridade. Considera-se estabelecido que a presença de colo curto no segundo trimestre da gravidez aumenta o risco para o parto prematuro e quanto menor o comprimento do colo uterino, 
menor a idade gestacional do parto. No entanto, é controverso na literatura o ponto de corte ideal do comprimento do colo uterino que permite a identificação das grávidas com risco aumentado para o parto prematuro. Os diversos estudos têm utilizado comprimentos que variam de $15 \mathrm{~mm}$ a 30 $\mathrm{mm}$. Porém, devido a esta ampla faixa, os resultados da sensibilidade também são muito diferentes, variando de 35 a $78 \%{ }^{5-8}$.

Em estudo envolvendo 2915 grávidas na $23^{a}$ e $24^{\text {a }}$ semana, foi obtido risco relativo para o parto prematuro de 9,4 quando o comprimento do colo uterino era menor que $22 \mathrm{~mm}^{7}$. Em outro estudo, também realizado no mesmo período gestacional, 2567 grávidas foram submetidas à ultra-sonografia transvaginal para avaliação do comprimento do colo uterino. Em 1,5\% das gestantes foi observado comprimento do colo igual ou menor que 15 $\mathrm{mm}$. Das grávidas que evoluíram para parto prematuro em idade gestacional inferior a 28 semanas, $86 \%$ tinham o comprimento do colo menor ou igual a $15 \mathrm{~mm}^{6}$.

Poucos estudos utilizaram a medida do comprimento do colo uterino no primeiro trimestre ${ }^{9-}$ 11. Avaliando o comprimento do colo uterino em grávidas com idade gestacional média de 11 semanas e 3 dias, foi observado comprimento médio de $46 \mathrm{~mm}$, sendo que o percentil 10 correspondeu a $40 \mathrm{~mm}$. A medida do comprimento do colo uterino abaixo do percentil $10(40 \mathrm{~mm})$ correlacionouse significativamente com o trabalho de parto prematuro antes da $30^{a}$ semana de gravide $z^{2}$.

A avaliação do colo uterino na gestação faz parte das estratégias de desenvolvimento de testes preditivos para o parto prematuro espontâneo. Um dos grandes problemas da Obstetrícia moderna é a incidência da prematuridade, que tem se mantido estável ao longo dos anos, apesar dos esforços desenvolvidos para sua prevenção. O parto prematuro ocorre em 6 a 10\% dos nascimentos, dependendo das características da população estudada ${ }^{12}$.

Em nosso meio não dispomos de estudos em população geral de grávidas e uma vez que a prematuridade tem etiopatogenia multifatorial, é importante conhecermos a influência do comprimento do colo uterino na prematuridade na nossa população. Desta maneira, neste estudo, buscamos avaliar a medida do comprimento do colo do útero por meio da ultra-sonografia transvaginal no primeiro e segundo trimestre da gravidez e correlacionar com a idade gestacional do parto.

\section{Pacientes e Métodos}

O estudo, prospectivo e longitudinal, envolveu inicialmente 641 gestantes do pré-natal do
Hospital das Clínicas da FMUSP no período de janeiro de 1998 a março de 1999. Os critérios de inclusão foram: gestação com feto único, datada pelo dia da última menstruação ou ultra-sonografia, quando havia dois exames compativeis até 20 semanas e não concordantes com a idade gestacional menstrual. Foram excluídas do estudo 18 grávidas que tiveram parto prematuro secundário a alguma indicação materna ou fetal (parto prematuro eletivo) ou aquelas das quais não obtivemos o resultado do parto $(n=56)$. As variáveis maternas analisadas foram: comprimento do colo uterino entre a $11^{\mathrm{a}}$ e a $16^{\mathrm{a}}$ semana e entre a $23^{\mathrm{a}}$ e a $24^{\mathrm{a}}$ semana.

Após assinatura de termo de consentimento informado, seguindo as normas da Comissão de Ética em Pesquisa do HCFMUSP, as pacientes foram admitidas no estudo.

A primeira avaliação do comprimento do colo uterino foi realizada entre a $11^{\mathrm{a}}$ e a $16^{\mathrm{a}}$ semana de gravidez. Após este exame, as gestantes foram agendadas para retornar entre a $23^{\text {a }}$ e a $24^{\text {a }}$ semana para realização de ultra-sonografia morfológica seguida da medida do comprimento do colo uterino pela via transvaginal.

A medida do comprimento do colo uterino foi realizada pela ultra-sonografia transvaginal segundo os passos descritos abaixo. Imediatamente antes do exame foi solicitado à gestante que esvaziasse a bexiga. Com a paciente em posição ginecológica, o transdutor transvaginal protegido com preservativo não lubrificado foi posicionado no terço externo da vagina para obtenção do corte sagital do colo uterino. O orifício interno e externo do colo, o canal e mucosa endocervical foram identificados. O comprimento do colo foi medido linearmente entre o orificio interno e o externo, delimitados pelo início e fim da mucosa endocervical. A duração do exame transvaginal foi de pelo menos 3 minutos para que as modificações, por vezes observadas durante o exame, fossem identificadas. Foi anotado sempre o menor comprimento de colo uterino observado durante este período. Foram utilizados os aparelhos da marca Toshiba, modelo Sonolayer 77 e Eccocce, com transdutor vaginal de $6 \mathrm{MHz}$ com $90^{\circ}$ de campo visual (modelo 601V).

Em relação ao resultado da gestação foi levada em consideração a idade gestacional do parto para análise estatística. O parto prematuro foi definido como aquele que se deu com idade gestacional inferior a 37 semanas. Classificamos como parto prematuro extremo o que ocorreu em idade gestacional menor que 33 semanas. O resultado da gestação foi correlacionado com a medida do comprimento do colo uterino no primeiro e segundo exame.

Em relação à idade gestacional do parto fo- 
ram constituídos dois grupos de estudo: grupo de partos prematuros, constituído pelas grávidas que evoluíram durante o período do estudo para parto prematuro, e grupo a termo, com aquelas gestantes que evoluiram com parto com idade gestacional maior ou igual a 37 semanas.

Para variáveis contínuas observamos valores minimos, máximos, cálculos de médias e desviospadrão, ao passo que para as variáveis classificatórias foram calculadas as freqüências absolutas e relativas. Para comparação entre as médias dos dois grupos foi utilizado o teste $t$ de Student. Para avaliar a eficácia de determinado comprimento do colo uterino na predição do parto prematuro foram utilizados os índices de sensibilidade, especificidade, taxa de falso-positivos, falsonegativos e acurácia. A hipótese de igualdade entre os momentos avaliados nos dois grupos estudados foi testada utilizando-se a estatística de Wilks com aproximação para estatística F. Para se obterem fatores preditivos da ocorrência de prematuros foi utilizada a técnica de regressão logística com processo de seleção stepwise. O nivel de significância utilizado para os testes foi de $5 \%$.

\section{Resultados}

Participaram inicialmente do estudo 641 grávidas, sendo o resultado do parto obtido em 585 casos $(91,3 \%)$. Do total de 585 grávidas, 44 (7,5\%) evoluiram para parto prematuro, das quais $18 \mathrm{ca}-$ sos foram classificados como prematuridade eletiva, portanto, excluídos da análise estatística.

A idade gestacional média dos partos foi de 39 semanas (variando de 26 semanas e 6 dias a 42 semanas e 2 dias). A incidência de partos prematuros espontâneos foi 4,6\% (26 casos), sendo $1,8 \%$ partos prematuros extremos (10 casos).

Do total de gestantes avaliadas entre a $11^{\text {a }}$ e a $16^{a}$ semana de gravidez, o resultado do parto foi obtido em 529 casos. A média do comprimento do colo, neste período da gestação, foi de $42,4 \mathrm{~mm}$ (variação de 25 a $74 \mathrm{~mm}$ ).

Das gestantes submetidas ao segundo exame, o resultado do parto foi obtido em 567 casos. O comprimento médio foi de $38,6 \mathrm{~mm}$ (variação de 6,6 $\mathrm{mm}$ a $57,1 \mathrm{~mm})$.

Os dois grupos de pacientes não mostraram diferença significante quanto ao comprimento do colo, quanto à avaliação entre a $11^{\mathrm{a}}$ e a $16^{\mathrm{a}}$ semana. A média dos comprimentos entre as que evoluíram para parto a termo foi de $42,7 \mathrm{~mm}$ e para as que tiveram parto prematuro foi de $40,6 \mathrm{~mm}$ ( $\mathrm{p}=0,2459$, teste $t$ de Student).

No entanto, a avaliação do comprimento do colo, entre a $23^{a}$ e a $24^{a}$ semana, mostrou diferença significativa entre os grupos de parto a termo e prematuro ( $\mathrm{p}=0,0001$, teste $t$ de Student). No grupo de parto a termo, o comprimento médio foi de $39,3 \mathrm{~mm}$ e, no grupo de partos prematuros, a média obtida foi de $26,7 \mathrm{~mm}$.

Em relação à prematuridade extrema, tanto o comprimento do colo uterino obtido entre a $11^{\mathrm{a}} \mathrm{e}$ a $16^{\mathrm{a}}$ semana de gravidez $(\mathrm{p}=0,0241)$ quanto entre a $23^{a}$ e a $24^{a}$ semana $(p=0,0007)$ foram significativamente diferentes nos grupos de parto a termo e prematuro (Tabela 1).

Tabela 1 - Valores de predição dos diferentes comprimentos do colo entre a $23^{\mathrm{a}}$ e a $24^{\mathrm{a}}$ semana de gravidez na predição do parto prematuro.

\begin{tabular}{lccccccc}
\hline Colo uterino & S & E & A & VPP & VPN & FP & FN \\
\hline$\leq 20 \mathrm{~mm}$ & 42,3 & 98,9 & 96,3 & 64,7 & 97,3 & 1,1 & 57,7 \\
$\leq 25 \mathrm{~mm}$ & 42,3 & 96,7 & 94,2 & 37,9 & 97,2 & 3,3 & 57,7 \\
$\leq 30 \mathrm{~mm}$ & 50,0 & 90,2 & 88,4 & 19,7 & 97,4 & 9,8 & 50.0
\end{tabular}

S: sensibilidade; E: especificidade; A: acurácia; VPP: valor preditivo positivo; VPN: valor preditivo negativo; FP: falso-positivos; FN: falso-negativos.

Do grupo de gestantes estudadas e com resultados completos da gestação, 529 pacientes foram submetidas à ultra-sonografia nos dois periodos do estudo (11-16 semanas e 23-24 semanas). A média do comprimento do colo uterino foi menor entre a $23^{\mathrm{a}}$ e a $24^{\mathrm{a}}$ semana do que entre a $11^{\mathrm{a}}$ e a $16^{a}$ semana e este encurtamento foi mais acentuado no grupo de gestantes que evoluíram para parto prematuro.

Nas gestantes com parto prematuro, a média do comprimento do colo uterino, entre a $11^{\text {a }} \mathrm{e}$ a $16^{\text {a }}$ semana, foi de $40,6 \mathrm{~mm}$ e, entre a $23^{\mathrm{a}}$ e a $24^{\mathrm{a}}$ semana, de $26,7 \mathrm{~mm}(\mathrm{p}=0,0001$, teste $t$ de Student).

Também comparando o comprimento do colo uterino nos dois momentos avaliados, no grupo de gestantes com parto a termo, foi observada diferença significativa $(42,7 \mathrm{~mm}$ e $39,3 \mathrm{~mm}$, respectivamente, $\mathrm{p}=0,0001$, teste de Wilks).

Desta forma, o comprimento médio do colo uterino, tanto no grupo de grávidas que evoluíram com parto prematuro ou a termo, diminuiu do exame realizado entre a $11^{\mathrm{a}}$ e a $16^{\mathrm{a}}$ semana para a $23^{a}-24^{a}$ semana de gravidez. O encurtamento do primeiro para o segundo exame, no entanto, foi mais acentuado no grupo de gestantes que evoluiram com parto prematuro (Figura 1).

A acurácia da medida do colo uterino para predição do parto prematuro foi avaliada pelos índices de sensibilidade, especificidade, valores preditivos positivo e negativo, falso-positivos e falso-negativos, para os comprimentos de até $20 \mathrm{~mm}$, até $25 \mathrm{~mm}$ e até $30 \mathrm{~mm}$ (Tabela 2). Em relação ao 
parto prematuro extremo, o pequeno número de casos não permitiu avaliar adequadamente a acurácia do comprimento do colo do útero para sua predição.

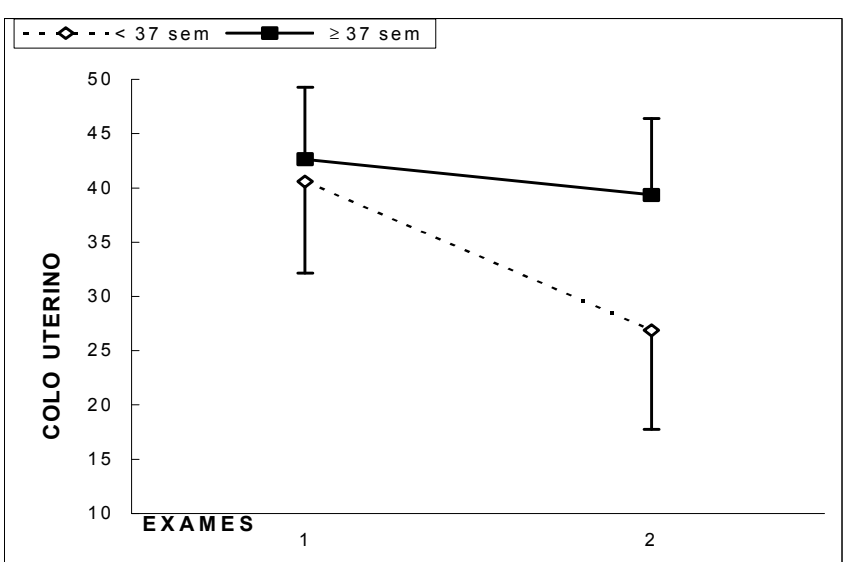

Figura 1 - Variabilidade do comprimento médio do colo uterino avaliado pela ultrasonografia transvaginal entre a $11^{\mathrm{a}}$ e a $16^{\mathrm{a}}$ semana, e entre a $23^{\mathrm{a}}$ e a $24^{\mathrm{a}}$ semana de gravidez, no grupo de gestantes com parto a termo e parto prematuro (análise de variâncias com medidas repetidas).

Tabela 2 - Médias do comprimento do colo uterino (em $\mathrm{mm}$ ) entre 11-16 semanas e entre 23-24 semanas de gestação, em relação à idade gestacional do nascimento.

\begin{tabular}{lccr}
\hline Idade gestacional & $<33$ sem & $<37$ sem & termo \\
\hline até 16 semanas & $37,6^{*}$ & 40,6 & $42,7^{*}$ \\
$23-24$ semanas & $22,4^{* *}$ & $26,7^{* *}$ & $39,3^{* *}$ \\
\hline$* \cdot p=0,0241^{* *} \cdot p<0,0001$ & &
\end{tabular}

$\mathrm{Na}$ Figura 2 demonstramos a porcentagem de partos prematuros em relação ao comprimento do colo uterino, menor ou igual a $20 \mathrm{~mm}$, ou maior que $20 \mathrm{~mm}$, entre 23 - 24 semanas de gestação.

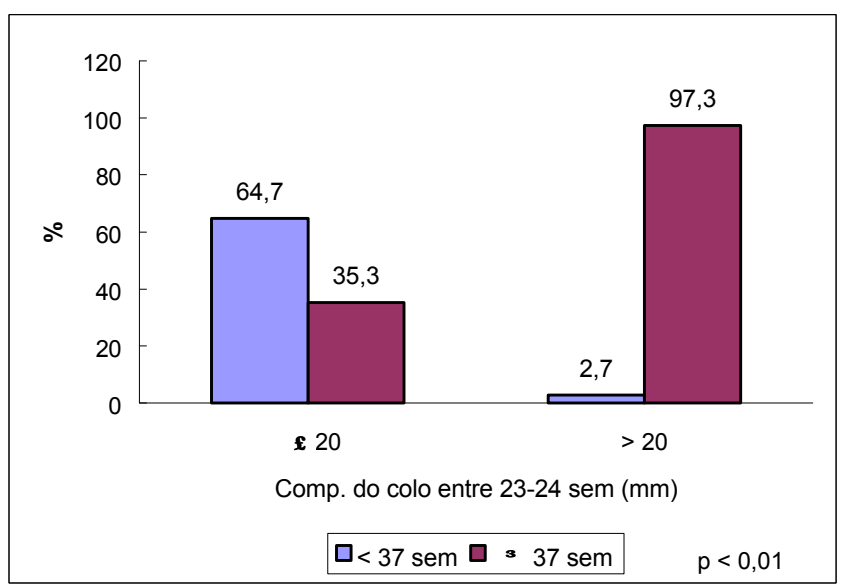

$<37$ sem: parto prematuro; = 37 sem: parto a termo; Comp: comprimento; $p<0,01$ : a porcentagem de partos prematuros entre as grávidas com comprimento de colo menor ou igual a $20 \mathrm{~mm}$ é estatisticamente diferente da porcentagem entre as grávidas com colo uterino maior que $20 \mathrm{~mm}$.

Figura 2 - Porcentagem de partos prematuros em relação ao comprimento do colo uterino entre a $23^{\mathrm{a}}$ e a $24^{\mathrm{a}}$ semana de gestação.
Com a técnica de regressão logística, o comprimento do colo uterino, entre a $23^{a}$ e a $24^{a}$ semana, foi identificado como fator de predição para o parto prematuro no presente trabalho.

\section{Discussão}

O comprimento do colo do útero constitui um dos fatores de predição do parto prematuro. Os colos mais curtos estão mais associados à prematuridade e a maior incidência de parto prematuro extremo (idade gestacional menor que 33 semanas) ${ }^{1,2}$.

A ultra-sonografia transvaginal é ótimo meio para a avaliação do colo uterino na gestação. Quando realizada pela via abdominal, os resultados não são bons, uma vez que, para a adequada visualização do colo uterino, é necessário o enchimento da bexiga, o que provoca um falso alongamento deste e fechamento do seu orifício interno ${ }^{13}$. A obesidade materna e o polo cefálico também dificultam a visualização do colo pela via abdominal. A avaliação pela via transvaginal não necessita de bexiga repleta e permite a visualização de toda a sua extensão, desde a porção interna até a externa ou vaginal, fornecendo o tamanho real do comprimento do colo uterino ${ }^{4,13}$.

No entanto, o exame do colo uterino pela via transvaginal também deve ser realizado com cautela, uma vez que aproximadamente $25 \%$ das medidas apresentam algum tipo de dificuldade de interpretação anatômica ou técnica ${ }^{14}$. Até o início do segundo trimestre, a diferenciação entre o segmento inferior do útero e o orificio interno do colo é uma das principais dificuldades anatômicas encontradas. A presença de contrações focais no segmento inferior também dificulta o exame, desfazendo transitoriamente o afunilamento, o que causa falsa medida de colo longo ${ }^{14}$. Isto leva a impressão de que, num curto período de tempo (3 a 5 minutos), a imagem ultra-sonográfica do colo se modifica, de ausência de dilatação do orificio interno do colo uterino, para a presença de afunilamento. Dificuldade técnica bastante comum é a avaliação dos colos com dilatação cervical ao exame manual. Deve-se associar, nestes casos, o corte transversal ao tradicional corte sagital do colo uterino como forma de melhor aferir sua dilatação ${ }^{14}$.

Avaliamos o comprimento do colo uterino pela ultra-sonografia transvaginal em dois momentos, sendo o primeiro entre a $11^{\mathrm{a}}$ e a $16^{\mathrm{a}}$ semana de gravidez e o outro entre a $23^{a}$ e a $24^{a}$ semana. Constatamos diminuição do comprimento médio do colo uterino do primeiro para o segundo exame, como descrito na literatura ${ }^{15-18}$. 
O comprimento do colo uterino avaliado pela ultra-sonografia transvaginal entre a $11^{\text {a }}$ e a $16^{a}$ semana não demonstrou ser eficaz na predição do parto prematuro, uma vez que o comprimento médio do colo não foi significativamente diferente entre as grávidas que evoluíram com parto prematuro e com parto a termo. No entanto, em relação ao parto com idade gestacional abaixo de 33 semanas, a média do comprimento do colo uterino foi de $37,5 \mathrm{~mm}$ contra $42,6 \mathrm{~mm}$ no grupo de parto com idade gestacional igual ou superior a 33 semanas. Este achado é compativel com outro estudo que observou aumento no risco para o parto com idade gestacional abaixo de 30 semanas, quando o comprimento do colo uterino encontrava-se abaixo do $10^{\circ}$ percentil $(40 \mathrm{~mm})$ no primeiro trimestre de gravide $z^{2}$. Estes achados nos levam a acreditar que a avaliação do colo uterino seja melhor na identificação das formas mais precoces de partos prematuros, podendo o encurtamento cervical já estar presente numa fase mais precoce da gravidez.

A medida do colo uterino entre a $23^{a}$ e a $24^{a}$ semana de gestação correlacionou-se com o parto prematuro. O comprimento médio do colo uterino, no grupo de gestantes com parto a termo, foi de $39,4 \mathrm{~mm}$ contra $26,7 \mathrm{~mm}$ no grupo de parto prematuro. Utilizando o comprimento do colo menor que $30 \mathrm{~mm}$, tivemos sensibilidade de $50 \%$ com valor preditivo positivo de $19,7 \%$ para o parto com menos de 37 semanas. Observamos que quanto menor o comprimento do colo uterino, menor a sensibilidade, porém maior a especificidade e o valor preditivo positivo, com menor número de falso-positivos, melhorando, desta forma, a acurácia do método. Em nosso estudo, para o comprimento de colo uterino menor ou igual a $20 \mathrm{~mm}$ a sensibilidade e o valor preditivo positivo para o parto prematuro (idade gestacional abaixo de 37 semanas) foram de 42,3 e 64,7\%, respectivamente, para uma taxa de falso-positivos de apenas 1,1\%. Em outro estudo envolvendo 3694 grávidas consideradas de baixo risco para prematuridade avaliadas entre a $18^{\mathrm{a}}$ e a $22^{\mathrm{a}}$ semana da gravidez, a sensibilidade e o valor preditivo positivo foram de 6 e 39\%, respectivamente, considerando o ponto de corte do colo uterino menor ou igual a $25 \mathrm{~mm}$. Estes valores são inferiores aos encontrados em nosso estudo e uma possível explicação para esta diferença é a presença de alguns exames em torno da $18^{\mathrm{a}}$ semana, o que é mais precoce do que o nosso. Assim sendo, estaria concordante com o nosso estudo no qual a avaliação do colo uterino até a $16^{a}$ semana de gravidez não foi efetiva na predição do parto prematuro.

Entretanto, a observação da relação do comprimento do colo uterino, no segundo trimestre de gravidez, com o parto prematuro, está em concordância com diversos estudos já relatados na literatura ${ }^{5,9,19-21}$, inclusive com outro estudo também realizado no nosso setor, entre gestantes com história prévia de prematuridade, ocasião em que foi observado um comprimento do colo menor nas gestantes que evoluíram para parto prematuro ${ }^{8}$.

A etiologia do parto prematuro é multifatorial e a identificação precoce das gestantes de risco para prematuridade seria o primeiro passo para a adequada prevenção.

Nesse estudo, o comprimento do colo uterino, entre a $23^{\mathrm{a}}$ e a $24^{\mathrm{a}}$ semana de gravidez, foi identificado como importante critério preditivo para o parto prematuro. Assim, acreditamos que a avaliação cervical nesta época da gestação deve ser oferecida para as grávidas como forma de avaliação dos riscos para prematuridade. De acordo com o presente estudo, assim como com outro realizado em nosso serviço em que o ponto de corte para aumento do risco para prematuridade também foi de $20 \mathrm{~mm}$ ou $\mathrm{menos}^{8}$, estabelecemos o seguinte protocolo: grávidas com comprimento do colo maior de $20 \mathrm{~mm}$ podem ser consideradas baixo risco para prematuridade desde que, após detalhada anamnese, sejam descartados outros fatores de risco para esta grávida. As grávidas com comprimento de colo uterino, menor ou igual a $20 \mathrm{~mm}$ são classificadas com alto risco para prematuridade e seguem protocolo específico assistencial, pelo qual são investigadas infecções vaginais e urinárias, solicitada diminuição da atividade física ou até mesmo o repouso. O uso da progesterona vaginal também é considerado, principalmente nos casos em que há antecedente de parto prematuro. Internamos a grávida sempre que haja sinais e sintomas sugestivos de trabalho de parto prematuro. Em nosso serviço, não realizamos a cerclagem cervical rotineira nos casos de colo curto.

Apesar dos achados correlacionando o colo curto com o parto prematuro, temos a convicção da necessidade de estudo com maior casuística, de preferência multicêntrico, com objetivo de melhor estabelecer o risco para o parto prematuro, levando-se em conta as alterações do comprimento do colo uterino.

\section{ABSTRACT}

Purpose: to evaluate, in the first and second trimesters of pregnancy, the correlation between cervical length and spontaneous preterm delivery.

Methods: cervical length was evaluated in 641 pregnant women between 11-16 weeks' and 23-24 weeks' gestation. 
Cervical assessment was performed by a transvaginal scan with the patient with empty bladder in a gynecological position. Cervical length was measured from the internal to the external os. The gestational age at delivery was correlated with the length of the cervix. To compare the means in groups of pregnant women who had a term or preterm delivery, we used Student's t test. Sensitivity, specificity, falsepositive and false-negative rates, and accuracy were calculated for cervical length of $20 \mathrm{~mm}$ or less, $25 \mathrm{~mm}$ or less and $30 \mathrm{~mm}$ or less in the prediction of preterm delivery.

Results: the measurement of cervical length, between 11 and 16 weeks of pregnancy, did not show any statistically significant difference on comparing women who had preterm and term delivery $(40.6 \mathrm{~mm}$ and $42.7 \mathrm{~mm}$, respectively, $p=0.2459$ ). However, the difference between the two groups at 23 to 24 weeks was significant $(37.3 \mathrm{~mm}$ in the group who delivered prematurely and $26.7 \mathrm{~mm}$ in the term group, $p=0.0001$, Student's t test).

Conclusion: there was no significant difference in cervical length, at 11 to 16 weeks, between pregnant women who had a preterm and term delivery. However, at 23 to 24 weeks, cervical length was significantly different between the two groups, and this measurement might be used as a predictor for prematurity.

KEY WORDS: Preterm delivery. Prematurity. Normal pregnancy. Uterine cervix length.

\section{Referências}

1. Romero R, Gonzalez R, Sepulveda W, et al. Infection and labor. VIII. Microbial invasion of the amniotic cavity in patients with suspected cervical incompetence: prevalence and clinical significance. Am J Obstet Gynecol 1992; 167:1086-91.

2. Zalar RW Jr. Early cervical length, preterm prelabor and gestational age at delivery. Is there a relationship? J Reprod Med 1998; 43:1027-33.

3. Hartmann K, Thorp JM Jr, McDonald TL, Savitz DA, Granados JL. Cervical dimensions and risk of preterm birth: a prospective cohort study. Obstet Gynecol 1999; 93:504-9.

4. Andersen HF. Ultrasound evaluation of the cervix and value of cervical cerclage. In: Elder MG, Romero $\mathrm{R}$, Lamont RF, editors. Preterm Labor. New York: Churchill Livingstone; 1997. p.165-84.

5. Murakawa H, Utumi T, Hasegawa I, Tanaka K, Fuzimori, R. Evaluation of threatened preterm delivery by transvaginal ultrasonographic measurement of cervical length. Obstet Gynecol 1993; 82:829-32.

6. Heath VC, Southall TR, Souka AP, Elisseou A, Nicolaides KH. Cervical length at 23 weeks of gestation: prediction of spontaneous preterm delivery. Ultrasound Obstet Gynecol 1998; 12:312-7.

7. Iams JD, Goldenberg RL, Meis PJ, et al. The length of the cervix and the risk of spontaneous premature delivery. N Engl J Med 1996; 334:567-72.
8. Yamasaki AA, Bittar RE, Fonseca ESB, Martinelli S, Sasaki S, Zugaib M. Prevenção do parto prematuro: emprego do toque vaginal e da ultra-sonografia transvaginal. Rev Bras Ginecol Obstet 1998; 20:350-6.

9. Berghella V, Tolosa JE, Kuhlman K, Weiner S, Bolognese RJ, Wapner RJ. Cervical ultrasonography compared with manual examination as a predictor of preterm delivery. Am J Obstet Gynecol 1997; 177:723-30.

10.Taipale P, Hiilesmaa V. Sonographic measurement of uterine cervix at 18-22 weeks' gestation and the risk of preterm delivery. Obstet Gynecol 1998; 92:902-7.

11.Guzman ER, Mellon C, Vintzileos AM, Ananth CV, Walters C, Gipson K. Longitudinal assessment of endocervical canal length between 15 and 24 weeks' gestation in women at risk for pregnancy loss or preterm birth. Obstet Gynecol 1998; 92:31-7.

12.Morrison JJ, Rennie JM. Clinical, scientific and ethical aspects of fetal and neonatal care at extremely preterm periods of gestation. $\mathrm{Br} \mathrm{J}$ Obstet Gynaecol 1997; 104:1341-50.

13. Okitsu O, Mimura T, Nakayama T, Aono T. Early prediction of preterm delivery by transvaginal ultrasonography. Ultrasound Obstet Gynecol 1992; 2:402-9.

14.Yost NP, Bloom ST, Twickler DM, Leveno KJ. Pitfalls in ultrasonic cervical length measurement for predicting preterm birth. Obstet Gynecol 1999; 93:510-6.

15.Iams JD. Cervical ultrasonography. Ultrasound Obstet Gynecol 1997; 10:156-60.

16.Guzman ER, Benito C, Hanley M. Sonography in the evaluation of the cervix during pregancy. Curr Opin Obstet Gynecol 1996; 8:99-105.

17.Kushnir O, Vigil DA, Izquierdo L, Schiff M, Curet LB. Vaginal ultrasonographic assessment of cervical length changes during normal pregnancy. Am J Obstet Gynecol 1990; 162:991-3.

18. Carvalho MHB, Brizot ML, Bittar RE, Miyadahira S, Zugaib M. Cervical length distribution in early pregnancy evaluated by transvaginal ultrasonography. Ultrasound Obstet Gynecol 1998; 12:11.

19.Rizzo G. Use ultrasound to predict preterm delivery: do not lose the opportunity. Ultrasound Obstet Gynecol 1996; 8:289-92.

20. Heath VC, Southall TR, Souka AP, Novakov A, Nicolaides KH. Cervical length at 23 weeks of gestation: relation to demographic characteristics and previous obstetric history. Ultrasound Obstet Gynecol 1998; 12:304-11.

21.Oliveira TA, Carvalho CMP, Souza E, et al. Avaliação do risco de parto prematuro: teste da fibronectina fetal e medida do colo uterino. Rev Bras Ginecol Obstet 2000; 22:633-9. 